\title{
ARTICLE
}

Received 25 May 2016 | Accepted 25 May 2017 | Published 17 Jul 2017

\section{High-order harmonic generation from the dressed autoionizing states}

\author{
M.A. Fareed ${ }^{1}$, V.V. Strelkov ${ }^{2,3}$, N. Thiré ${ }^{1}$, S. Mondal ${ }^{1,4}$, B.E. Schmidt1,5, F. Légaré1 \& T. Ozaki ${ }^{1}$
}

In high-order harmonic generation, resonant harmonics $(\mathrm{RH})$ are sources of intense, coherent extreme-ultraviolet radiation. However, intensity enhancement of $\mathrm{RH}$ only occurs for a single harmonic order, making it challenging to generate short attosecond pulses. Moreover, the mechanism involved behind such $\mathrm{RH}$ was circumstantial, because of the lack of direct experimental proofs. Here, we demonstrate the exact quantum paths that electron follows for $\mathrm{RH}$ generation using tin, showing that it involves not only the autoionizing state, but also a harmonic generation from dressed-AIS that appears as two coherent satellite harmonics at frequencies $\pm 2 \Omega$ from the $\mathrm{RH}$ ( $\Omega$ represents laser frequency). Our observations of harmonic emission from dressed states open the possibilities of generating intense and broadband attosecond pulses, thus contributing to future applications in attosecond science, as well as the perspective of studying the femtosecond and attosecond dynamics of autoionizing states.

\footnotetext{
${ }^{1}$ Institut national de la recherche scientifique-Centre Energie Matériaux Telécommunications, 1650 Lionel-Boulet, Varennes, Québec, Canada J3X $1 S 2$. ${ }^{2}$ A. M. Prokhorov General Physics Institute of RAS, Vavilova street 38, 119991 Moscow, Russia. ${ }^{3}$ Moscow Institute of Physics and Technology (State University), 141700 Dolgoprudny, Moscow Region, Russia. ${ }^{4}$ ELI-ALPS, ELI-Hu Kft., Dugonics ter 13, H-6720 Szeged, Hungary. ${ }^{5}$ few-cycle, Inc., 2890 Rue de Beaurivage, Montreal, Quebec, Canada H1L 5W5. Correspondence and requests for materials should be addressed to T.O. (email: ozaki@emt.inrs.ca).
} 
igh-order harmonic generation (HHG) is an excellent tabletop source of coherent extreme ultraviolet (XUV) and soft X-ray radiation. As high-order harmonics are intrinsically generated in attosecond bursts, it also opens a new domain of attosecond science ${ }^{1-4}$. More recently, high-harmonic spectroscopy is developing as a unique tool for studying the electronic structure of atoms and molecules ${ }^{5,6}$. The HHG process from most nonlinear media is well explained by the three-step model $^{7}$. However, for some media, the intensity of a specific harmonic order could be enhanced by one to two orders of magnitude compared with the neighbouring harmonics, which are generated owing to conventional three-step process. This has been observed when the wavelength of a harmonic is in close proximity to an autoionizing resonance of the nonlinear medium $^{8,9}$. Major efforts up to now have been devoted to increasing the photon flux of these resonant harmonics $(\mathrm{RH})^{8,10,11}$. However, there have only been a few experiments that were aimed for better understanding of the physics involved behind this $\mathrm{RH}$ generation. These experiments have only shown that the $\mathrm{RH}$ intensity depends strongly on the ellipticity of the driving laser ${ }^{11}$, and follows phase matching conditions similar to other conventional gas harmonics ${ }^{10}$.

There have also been theoretical efforts aimed at better understanding the physics behind $\mathrm{RH}$ generation ${ }^{12-17}$. Different models have shown that the increase of the RH intensity can be attributed to the macroscopic processes ${ }^{13,14}$ (to improved phase matching of the $\mathrm{RH}$ generation) or to the microscopic ones ${ }^{15-19}$. In the latter direction, several different yet-to-be-verified theoretical models have been proposed using bound-bound ${ }^{15}$, bound-continuum transitions ${ }^{16}$ and the electronic motion in the continuum ${ }^{17}$ to explain the mechanism of $\mathrm{RH}$ generation. In particular, the four-step model, proposed by Strelkov ${ }^{17}$ has demonstrated an agreement with several experimental observations $s^{20,21}$. In this model, steps 1 and 2 are the same as for the three-step model. However, in step 3, the electron accelerated in the continuum is trapped by the parent ion, so that the system lands in the autoionizing state (AIS), and in step 4 relaxes to the ground state by emitting the harmonic photon. Experimentally observed intensity enhancements of $\mathrm{RH}$ have been compared with theory for several materials ${ }^{17}$, and the relative phase between $\mathrm{RH}$ and non- $\mathrm{RH}$ have been experimentally measured and compared with theory ${ }^{18}$. However, intensity and phase measurements of the harmonics are still indirect evidence of the four-step model, and thus there currently lacks direct and concrete evidence that the AIS is involved in RH generation.

In this work, we study the electron quantum paths in the vicinity of AIS with mid-IR tunable driving fields. Our results allow to unambiguously clarify the mechanism involved in $\mathrm{RH}$ generation. The present study shows that $\mathrm{RH}$ generation process involves the AIS for coherent harmonic emission at resonant energy via the microscopic response. Further, it has been revealed that the RH generation process involves the dressed-AIS for coherent harmonic emission at frequencies $\pm 2 \Omega$ from the RH frequency ( $\Omega$ represents laser frequency). As $\mathrm{RH}$ is an excellent candidate as a source for intense harmonics ${ }^{8,9}$, the involvement of dressed states in HHG opens the perspective to expand the bandwidth of $\mathrm{RH}$ (for example, we can generate harmonics at $\pm 2 \Omega, \pm 4 \Omega$, and above frequencies), thus opening the possibility to generate intense and ultrashort attosecond pulses, useful for the applications of attosecond science ${ }^{1,2}$. The direct involvement of AIS in RH generation via microscopic response will also provide opportunities to study emission dynamics of AIS with ultrafast lasers. For example, we can extract information about the absolute time that the electron stays in the AIS before emission, study electron-electron interaction, and interference of $\mathrm{RH}$ and direct harmonic at attosecond time scale by driving

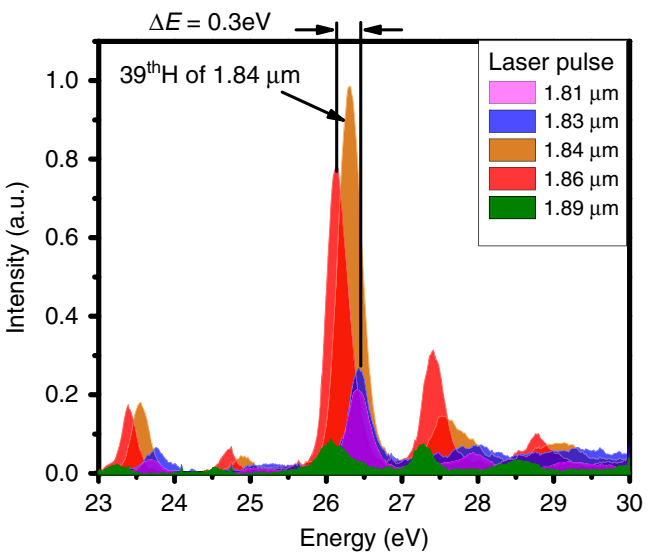

Figure 1 | High-order harmonic spectra with tunable driving laser. The extreme-ultraviolet spectra measured at different driving laser wavelengths ranging from $1.81 \mu \mathrm{m}$ to $1.89 \mu \mathrm{m}$. Maximum harmonic intensity is observed for a pulse centred at $1.84 \mu \mathrm{m}$, as this wavelength is exactly 39-photon resonant with the $4 d^{10} 5 s^{2} 5 p \rightarrow 4 d^{9} 5 s^{2} 5 p^{2}$ transition of $\mathrm{Sn}^{+}$. The harmonic intensity then decreases rapidly as the central driving laser wavelength is detuned. The driving laser intensity for these spectra is maintained at $\sim 1.3 \times 10^{14} \mathrm{~W} \mathrm{~cm}^{-2}$.

harmonics using pulses with certain duration, as the electron stays for a short time in the AIS. Further, harmonic emission from virtual quantum states will provide opportunities to understand the nature of virtual states and their influence on the physical systems both in physics and chemistry, hence providing better control on the systems and roots to find new applications.

\section{Results}

RH response with tunable driving wavelengths. We have observed variation in the intensity of the $\mathrm{RH}$ with the change in the central wavelength of the driving laser. As the driving laser used in this experiment is broadband $(\sim 100 \mathrm{~nm})$, we find that there is a certain wavelength range in which intensity enhancement of the RH could be observed ${ }^{11,20}$. We show in Fig. 1 the harmonic spectra from the $\mathrm{Sn}^{+}$around the AIS resonance, when the central wavelength of the driving laser is varied from 1.81 to $1.89 \mu \mathrm{m}$. The intensity of the RH is maximum for driving laser at $1.84 \mu \mathrm{m}$ central wavelength, as this wavelength is exactly 39-photon resonant with the $26.3 \mathrm{eV}$ autoionizing resonance of $\mathrm{Sn}^{+}$. As we increase the detuning of the driving laser wavelength from this resonance, the $\mathrm{RH}$ intensity decreases. Only weak $\mathrm{RH}$ is observed at laser wavelengths of 1.81 and $1.89 \mu \mathrm{m}$, which disappears completely at 1.80 and $1.90 \mu \mathrm{m}$ wavelengths. Therefore, these results show that when using driving lasers of $\sim 100 \mathrm{~nm}$ bandwidth, the $\mathrm{RH}$ of $\mathrm{Sn}^{+}$responds well for at least $\pm 30 \mathrm{~nm}$ of bandwidth, around the resonant wavelength of $\sim 1.84 \mu \mathrm{m}$.

Microscopic response of $\mathbf{R H}$. Our results allow defining if the observed enhancement of the $\mathrm{RH}$ intensity is due to the macroscopic or the microscopic processes. In Fig. 1, we observe that when harmonic is close to the resonance, the harmonic intensity is enhanced in the spectral region of $\sim 0.3 \mathrm{eV}$. The width of this spectral region is close to the width of the $4 d^{9} 5 s^{2} 5 p^{2}$ (1D) ${ }^{2} \mathrm{D}_{5 / 2}$ state of $\mathrm{Sn}^{+}(0.16 \mathrm{eV})$. The former is broader because of the non-zero harmonic line width and transition line broadening owing to AIS photoionization. The width of the enhancement region equal to $0.3 \mathrm{eV}$ was found numerically ${ }^{22}$, although much shorter driving wavelength was used in these calculations. 


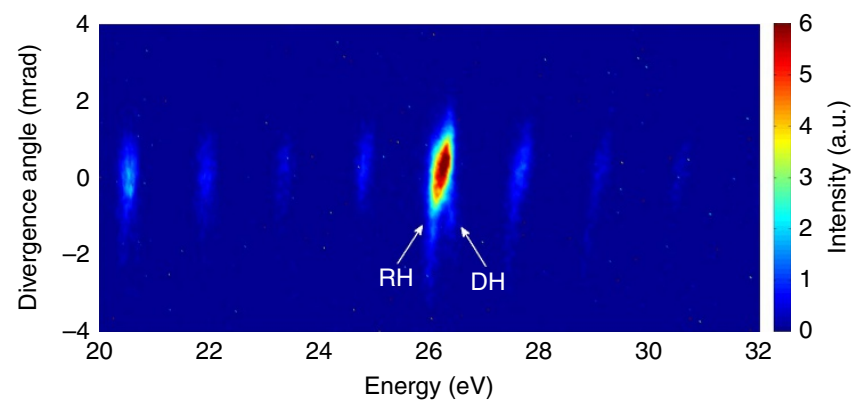

Figure 2 | Overlap of resonant harmonic and direct harmonic at resonant energy. High-order harmonic spectrum of $\mathrm{Sn}^{+}$at $1.75 \mu \mathrm{m}$ driving laser wavelength, showing that the stronger resonant harmonic $(\mathrm{RH})$ and relatively weak direct harmonic $(\mathrm{DH})$ are overlapped with each other at energy $\sim 26.3 \mathrm{eV}$. This spectrum is recorded at laser intensity of $\sim 1.43 \times 10^{14} \mathrm{~W} \mathrm{~cm}^{-2}$.

This shows that the enhancement in our conditions is due to the microscopic response enhancement and not due to the macroscopic effects because the region where the phase matching could be improved by the resonance is typically narrower than the resonance width, and the centre of this region is shifted with respect to the transition frequency. Moreover, we see that the harmonic below the resonance one is suppressed, in agreement with the microscopic response ${ }^{22}$. This effect was not previously observed in the HHG because the frequency range between the harmonics was larger due to higher driving frequency ${ }^{20}$.

Splitting of the harmonic spectral line. More features observed under small detuning from the resonance can be seen in Fig. 2. It shows a typical high-order harmonic spectrum from $\mathrm{Sn}^{+}$for driving lasers at $1.75 \mu \mathrm{m}$ wavelength, which is in 37-photon resonance with the $26.3 \mathrm{eV}$ autoionizing resonance ${ }^{11}$. We see that around energies of $26 \mathrm{eV}$ there are actually two close peaks. The frequency of the more intense one is the transition frequency, whereas the frequency of the other is the 37th harmonic frequency. Such harmonic line splitting was theoretically predicted in ref. 18. This theory, however, assumes initial coherent superposition of states of the generating particle. Further theoretical study of the splitting in more realistic situation of initial population only in the ground state was done in ref. 22. This theory shows, in particular, that the line shape of the harmonic near the resonance is a product of the harmonic line that would be emitted in the absence of the resonance, and the factor that is unity far from the resonance and has a sharp peak at the resonant frequency. For certain detunings of the harmonic wavelength from the resonance, this product results in a double-peak structure, where one peak is close to the harmonic frequency and the other is close to the resonance frequency.

This splitting is an evidence of the coherent emission owing to the following processes: the peak at the transition frequency is emitted due to the four-step process, where the wing of the harmonic line overlapping with the transition frequency is resonantly enhanced, and the peak at the exact harmonic frequency is emitted due to the three-step process, where the electrons from the continuum directly recombine to the ground state, as illustrated in Fig. 2. For brevity, we denote these peaks as $\mathrm{RH}$ and direct harmonic (DH), respectively.

Thus, by using a wavelength tunable driving laser and taking advantage of the wavelength locked $\mathrm{RH}$ emission (discussed above), we demonstrate that the $\mathrm{DH}$ could be
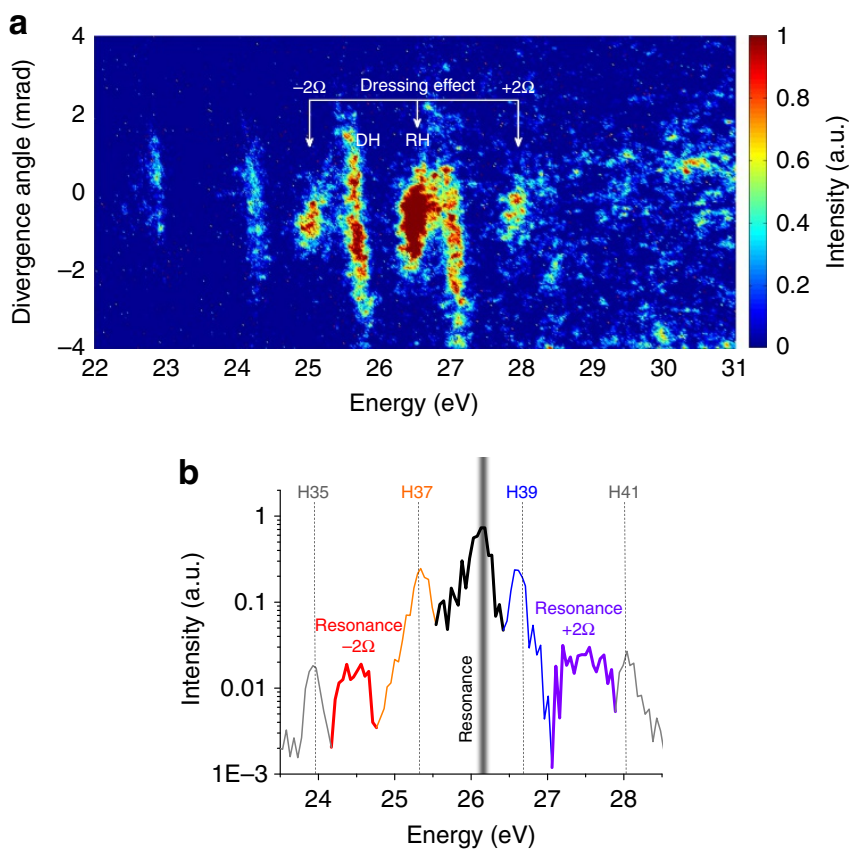

Figure 3 | Separation of resonant harmonic and direct harmonic at detuned driving laser. (a) High-order harmonic spectrum of $\mathrm{Sn}^{+}$using a driving laser with $1.785 \mu \mathrm{m}$ central wavelength. This spectrum demonstrates that the resonant harmonic $(\mathrm{RH})$ and the direct harmonic (DH) can be separated by detuning the driving laser wavelength. In this spectrum, two additional coherent harmonics are observed at $\pm 2 \Omega$ around the $\mathrm{RH}$, which are generated from the dressed autoionizing state (AIS). This spectrum is recorded at a driving laser intensity of $\sim 1.4 \times 10^{14} \mathrm{~W} \mathrm{~cm}^{-2}$.

(b) The harmonic spectrum calculated via 3D time-dependent Schrödinger equation for a model $\mathrm{Sn}^{+}$ion in the laser field, showing good agreement with experimental observations.

separated from the $\mathrm{RH}$ by carefully detuning the driving laser wavelength from the resonant wavelength.

Harmonic emission from the dressed-AIS. Figure 3 a shows the spectrum in the vicinity of the transition frequency for $1.785 \mu \mathrm{m}$ driving wavelength. When the central driving laser wavelength is tuned from 1.75 to $1.785 \mu \mathrm{m}$, the $\mathrm{RH}$ and the $\mathrm{DH}$ are separated from each other. Moreover, with this off-resonance detuning, we reveal a never-considered feature of high-order harmonics. Namely, we demonstrate in Fig. 3a that when the driving laser wavelength is detuned, two additional harmonics appear at energies $\sim 25.0 \mathrm{eV}$ and $\sim 27.9 \mathrm{eV}$. These satellite harmonics are located exactly at $\pm 2 \Omega$ around the $R H$, where $\Omega$ is the photon energy of the driving laser. One can also notice that the divergence of both satellite harmonics is close to the divergence of the RH. We explain these observations as due to the generation of harmonics from the dressed AIS.

Theoretical calculations. We perform simulations to model our experimental observations. Our theoretical study is based on the numerical solution of the 3D time-dependent Schrödinger equation for a model $\mathrm{Sn}^{+}$ion in an external laser field. The details of the theoretical approach are described in the Supplementary Information. Figure $3 b$ shows the calculated XUV spectrum near the resonance of tin. In this spectrum, we see the usual 37th (Orange line) and the 39th (Blue line) harmonics, and additional peaks in between, in agreement with the experimental results. The position of the additional peak at the red-side of the 


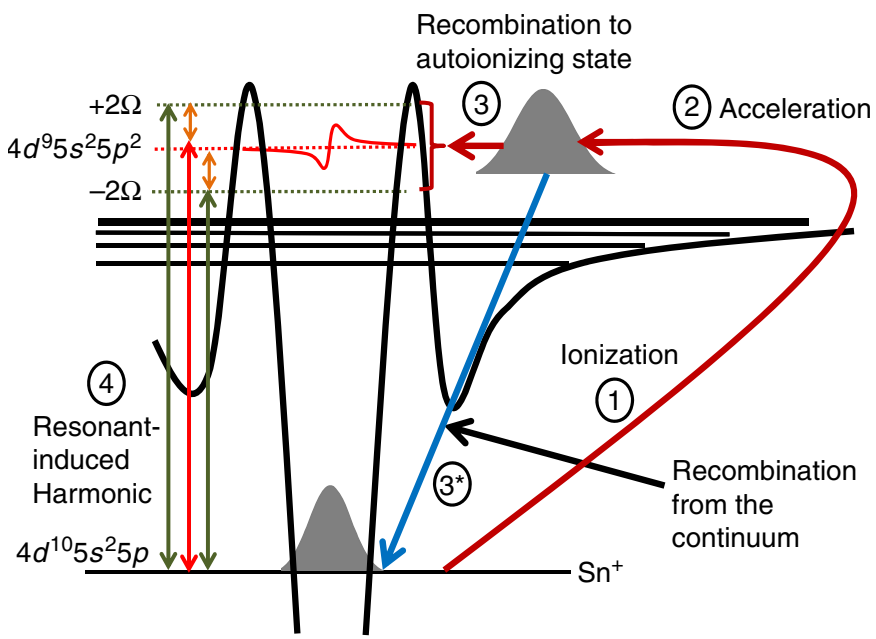

Figure 4 | Schematic illustrations of electron quantum paths. Schematic diagram describing the four-step model of HHG from dressed AIS of $\mathrm{Sn}^{+}$. When high-intensity laser pulses $\left(\sim 10^{14} \mathrm{~W} \mathrm{~cm}^{-2}\right)$ interact with $\mathrm{Sn}^{+}$, an electron is detached from the ground state (Step 1). This electron is then accelerated in the continuum by the laser field (Step 2). In the third step, the continuum electron has two possible paths to follow. It can either recombine directly to the ground state (Step $3^{\star}$ ) and generate $\mathrm{DH}$, or it can be trapped so that the system finds itself in the AIS or dressed AIS (Step 3) and then relaxes to the ground state and emits the RH (Step 4). In the latter scenario, three coherent $\mathrm{RHs}$ are generated.

39th harmonic (Black line) is close to the exact resonance. This splitting of the harmonic line near the resonance can be well-understood within the analytical theory ${ }^{22}$ as was described above. We also see that there are additional peaks (satellites) on the red side of the 37 th (red line) and the 41st (violet line) harmonics. Gabor analysis is used to understand the origin of these peaks. The complete details of this analysis are given in the Supplementary Information. In short, the calculations indicate that opposite to the usual harmonics these satellites are emitted only when the central RH is emitted, thus when the AIS is populated and when the laser field is on. The AIS dressing naturally takes place only within the laser pulse, so these results indicate that the satellites emission involves the resonance with the dressed AIS. Thus, the calculations confirm that the satellite harmonics experimentally observed in Fig. 3a at $\pm 2 \Omega$ around the $\mathrm{RH}$ are generated from the dressed-states of the autoionizing resonance.

RH generation process. Previous studies of $\mathrm{RH}$ generation were focused at a single state (the AIS for the case of $\mathrm{Sn}^{+}$), which is in the multiphoton resonance with the driving laser ${ }^{11,20}$. However, our experimental observations show that $\mathrm{RH}$ generation can involve three different states: the actual AIS and the two dressed states located at $\pm 2 \Omega$ around the resonant AIS. At the third step of the four-step model the system finds itself in one of these states and then, at the fourth step, it recombines to the ground state and emits one of the three RHs. This complete mechanism of $\mathrm{RH}$ generation is illustrated in Fig. 4. It is obvious that when the driving wavelength corresponds to the exact multi-photon resonance, the harmonics from these dressed states overlap with the neighbouring non- $\mathrm{RH}$, and thus the two are difficult to differentiate (Fig. 2). However, these satellite harmonics can be distinguished from each other when the driving wavelength is detuned. Thus, this observation shows that the driving wavelength detuning from the resonance allows to observe the very pronounced separation of $\mathrm{RH}$ and $\mathrm{DH}$, as well as the spectral peaks generated due to the dressed AIS.

Note that very pronounced enhancement of the harmonics neighbour to the resonant one was found theoretically in ref. 19 . This theory considers a coherent superposition of ground and excited states formed by pumping by the $\mathrm{RH}$, and thus assumes quite high intensity of this harmonic. The moderate intensity of $\mathrm{RH}$ in our experiments (in general, comparable to the one of the $\mathrm{DH}$ ) prevents the direct application of this theory for their interpretation. However, our explanation of the process, presented in Fig. 4, can be understood as well in the terms of theories ${ }^{18,19}$, involving coherent superposition of states: the coherent superposition of the ground state and AIS appears as a result of the rescattering (as it was suggested in ref. 17), and then interacts with the laser field, demonstrating features predicted in ref. 19.

Our observations provide the accurate information of the quantum path that the electron follows in the vicinity of the AIS for $\mathrm{RH}$ generation. We have shown that for $\mathrm{RH}$ generation the electron quantum path is perturbed not only from the AIS but also from the dressed states, resulting in three coherent harmonic generation. In high-order harmonic attosecond science, pump-probe absorption spectroscopy has been recently started to study the light-matter interaction with dressed $\mathrm{AIS}^{23,24}$. However, the propagation and emission dynamics of the electron from dressed AIS have been highly ignored. Our observation of satellite harmonics from dressed AIS shows that the AIS dressing by the laser field should be taken into account to describe also the emission processes during the light-matter interaction for elements containing AIS. As AIS exist in different materials ${ }^{25}$, the present work will be applicable in general to study the influence of dressed states on electron quantum path in all these materials. As dressed states respond within the driving laser pulse, the nonlinear properties of the dressed AIS will provide the opportunity to study and control electronic motion at fast timescales, which will be an advantage as electrons remain in the AIS for only a few 100 attoseconds to a few femtoseconds.

\section{Methods}

Experimental set-up. The schematic diagram of the experimental setup used for HHG from laser-ablated plumes is shown in the Supplementary Fig. 1. The complete details of the mid-infrared laser source that was used for HHG can be found in ref. 26. In short, an uncompressed Ti:sapphire laser beam with a repetition rate $100 \mathrm{~Hz}$, pulse duration of $\sim 210$ ps and a central wavelength of $\sim 0.8 \mu \mathrm{m}$ is divided into two beams. The first beam, called the prepulse (PP), is focused on to a solid tin target to generate low-density plume, which will be the nonlinear medium for harmonic generation. The PP intensity is maintained at $\sim 10^{10} \mathrm{~W} \mathrm{~cm}^{-2}$ over a circular spot of $\sim 200 \mu \mathrm{m}$ in diameter. This intensity is used to ablate a plume from the target surface, placed inside a vacuum chamber having pressure $\sim 10^{-5}$ Torr. The second beam, called the main pulse (MP), is compressed to $\sim 42$ fs and then seeded to an optical parametric amplifier

(OPA; HE-TOPAS) for nonlinear frequency conversion. The output of this optical parametric amplifier, with energy $\sim 750 \mu \mathrm{J}$ and pulse duration $\sim 51 \mathrm{fs}$, is then subsequently amplified with an amplification stage. This laser system is capable of producing $10 \mathrm{~mJ}$ of energy at $1.8 \mu \mathrm{m}$ wavelength. The wavelength of these pulses are tuned close to the multiphoton resonance and then used for HHG, after a certain delay of $45 \mathrm{~ns}$ from the plume ablation. These high-order harmonics are then sent to a soft X-ray spectrometer, consisting of a flat-field Hitachi grating $(1,200$ lines per $\mathrm{mm})$ and a multi-channel plate (MCP) followed by a phosphor screen. Finally, the harmonic spectrum detected by the MCP is then captured by a 16-bit camera (CMOS, PCO-edge).

Coherence. When AIS are involved in RH generation, the common question that arises is whether RH emission is coherent or it is a spontaneous emission from the AIS. The coherent emission of RH can be checked simply by looking into the divergence of the harmonics on the MCP (Supplementary Fig. 2). The high-order harmonics have well-collimated emission that propagates collinearly with the driving laser field. However, spontaneous atomic or ionic transitions are emitted in $4 \pi$ direction, and thus cover the complete width of the slit $(1 \mathrm{~cm})$ of the XUV spectrometer. In reality, this emission angle is limited by the size of the slit, which is $\sim 11 \mathrm{mrad}$. Therefore, harmonic emission can easily be differentiated from the 
spontaneous emission, as can be seen in Supplementary Fig. 2. Further, the divergence of the $\mathrm{RH}$ is observed to be much smaller than the divergence of the non-RH, generated with the three-step process (Fig. 3a). This is because the RH of $\mathrm{Sn}^{+}$has well collimated emission owing to better phase matching than the harmonics generated with the three step process. The low divergence of the $\mathrm{RH}$ indicates that $\mathrm{RH}$ propagates with the non- $\mathrm{RH}$ in the same direction. The coherent $\mathrm{RH}$ emission occurs because of the short lifetime of the AIS involved in $\mathrm{RH}$ generation. The lifetime of the resonant state can be estimated with the line width of the AIS. The line width of the $4 d^{9} 5 s^{2} 5 p^{2}$ (1D) ${ }^{2} \mathrm{D}_{5 / 2}$ state is $\Gamma=160 \mathrm{meV}$, which corresponds to the life time of $\sim 4.1 \mathrm{fs}(\tau=\hbar / \Gamma)$. The similarity in divergence observed for the satellite harmonics further confirms the coherent emission of the RHs. Further information of the coherent RH emission can be found in ref. 8

Data availability. The data sets generated during and/or analysed during the current study are available from the corresponding author on reasonable request.

\section{References}

1. Krausz, F. \& Ivanov, M. Attosecond physics. Rev. Mod. Phys. 81, 163-234 (2009).

2. Corkum, P. \& Krausz, F. Attosecond science. Nat. Phys 3, 381-387 (2007)

3. Chini, M., Zhao, K. \& Chang, Z. The generation, characterization and applications of broadband isolated attosecond pulses. Nat. Photonics $\mathbf{8}$, 178-186 (2014).

4. Takahashi, E. J. Attosecond nonlinear optics using gigawatt-scale isolated attosecond pulses. Nat.Commun 4, 2691 (2013).

5. Shiner, A. D. et al. Probing collective multi-electron dynamics in xenon with high-harmonic spectroscopy. Nat. Phys 7, 464-467 (2011).

6. Itatani, J. et al. Tomographic imaging of molecular orbitals. Nature 432, 867-871 (2004).

7. Corkum, P. Plasma perspective on strong field multiphoton ionization. Phys. Rev. Lett. 71, 1994-1997 (1993).

8. Rothhardt, J. et al. Enhancing the macroscopic yield of narrow-band high-order harmonic generation by Fano resonances. Phys. Rev. Lett. 112, 233002 (2014).

9. Ganeev, R. A., Suzuki, M., Baba, M., Kuroda, H. \& Ozaki, T. Strong resonance enhancement of a single harmonic generated in the extreme ultraviolet range. Opt. Lett. 31, 1699-1701 (2006).

10. Rosenthal, N. \& Marcus, G. discriminating between the role of phase matching and that of the single-atom response in resonance plasma-plume high-order harmonic generation. Phys. Rev. Lett. 115, 133901 (2015).

11. Suzuki, M., Baba, M., Ganeev, R. A., Kuroda, H. \& Ozaki, T. Anomalous enhancement of a single high-order harmonic by using a laser-ablation tin plume at $47 \mathrm{~nm}$. Opt. Lett. 31, 3306-3308 (2006).

12. Milosevic, D. B. \& Becker, W. Role of long quantum orbits in high-order harmonic generation. Phys. Rev. A 66, 063417 (2002).

13. Elouga-Bom, L.-B., Bouzid, F., Vidal, F., Kieffer, J.-C. \& Ozaki, T. Correlation of plasma ion densities and phase matching with the intensities of strong single high-order harmonics. J. Phys. B 41, 215401 (2008).

14. Ganeev, R. A. et al. Comparative studies of resonance enhancement of harmonic radiation in indium plasma using multicycle and few-cycle pulses. Phys. Rev. A 88, 033838 (2013).

15. Faria, C. F. D. M., Kopold, R., Becker, W. \& Rost, J. M. Resonant enhancements of high-order harmonic generation. Phys. Rev. A 65, 023404 (2001).

16. Taïeb, R., Véniard, V., Wassaf, J. \& Maquet, A. Roles of resonances and recollisions in strong-field atomic phenomena. II. High-order harmonic generation. Phys. Rev. A 68, 033403 (2003).

17. Strelkov, V. Role of autoionizing state in resonant high-order harmonic generation and attosecond pulse production. Phys. Rev. Lett. 104, 123901 (2010).

18. Milosevic, D. B. Theoretical analysis of high-order harmonic generation from a coherent superposition of states. J. Opt. Soc. Am. B 23, 308-317 (2006).
19. Milosevic, D. B. High-energy stimulated emission from plasma ablation pumped by resonant high-order harmonic generation. J. Phys. B 40, 3367-3376 (2007).

20. Haessler, S. et al. Phase distortions of attosecond pulses produced by resonance-enhanced high harmonic generation. New J. Phys. 15, 013051 (2013).

21. Ganeev, R. A. High-order harmonic generation in laser plasma plumes (World Scientific, 2013).

22. Strelkov, V. V., Khokhlova, M. A. \& Shubin, N. Y. High-order harmonic generation and Fano resonances. Phys. Rev. A 89, 53833 (2014).

23. Wu, M., Chen, S., Camp, S., Schafer, K. J. \& Gaarde, M. B. Theory of strongfield attosecond transient absorption. J. Phys. B 49, 062003 (2016).

24. Chini, M. et al. Sub-cycle oscillations in virtual states brought to light. Sci. Rep 3, 1105 (2013).

25. Becker, U. \& Shirley, D. VUV and Soft X-Ray Photoionization (Plenum, 1996).

26. Thiré, N. et al. $10 \mathrm{~mJ} 5$-cycle pulses at $1.8 \mu \mathrm{m}$ through optical parametric amplification. Appl. Phys. Lett. 106, 091110 (2015).

\section{Acknowledgements}

The authors acknowledge the financial support from the Fonds de recherche du Québec-Nature et technologies (FRQNT), the Natural Sciences and Engineering Research Council of Canada (NSERC), and the Canada Foundation for Innovation-Major Science Initiatives. Theoretical studies were supported by RSF (grant 16-12-10279).

\section{Author contributions}

M.A.F. designed the experiment with T.O. Moreover, N.T., B.E.S. and F.L. provided the laser source. M.A.F. performed the experiments with S.M. and N.T. Further, M.A.F. analysed the results and V.V.S. carried out simulations. M.A.F. and V.V.S. wrote the manuscript with T.O. All authors contributed to the discussion of the experimental data and the manuscript.

\section{Additional information}

Supplementary Information accompanies this paper at http://www.nature.com/ naturecommunications

Competing interests: The authors declare no competing financial interests.

Reprints and permission information is available online at http://npg.nature.com/ reprintsandpermissions/

How to cite this article: Fareed, M. A. et al. High-order harmonic generation from the dressed autoionizing states Nat. Commun. 8, 16061 doi: 10.1038/ncomms16061 (2017).

Publisher's note: Springer Nature remains neutral with regard to jurisdictional claims in published maps and institutional affiliations.

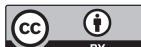

Open Access This article is licensed under a Creative Commons Attribution 4.0 International License, which permits use, sharing, adaptation, distribution and reproduction in any medium or format, as long as you give appropriate credit to the original author(s) and the source, provide a link to the Creative Commons license, and indicate if changes were made. The images or other third party material in this article are included in the article's Creative Commons license, unles indicated otherwise in a credit line to the material. If material is not included in the article's Creative Commons license and your intended use is not permitted by statutory regulation or exceeds the permitted use, you will need to obtain permission directly from the copyright holder. To view a copy of this license, visit http://creativecommons.org/ licenses/by/4.0/

(C) The Author(s) 2017 